\title{
Comparative Study on the Outcomes of Elective-Start versus Urgent-Start Peritoneal Dialysis Catheter Placement
}

\author{
Ahmed Kamel Abdel Aal $\mathbb{D}^{1},{ }^{1}$ Khalid Mahmoud, ${ }^{2}$ Amr Soliman Moustafa, ${ }^{3,4}$ \\ Noha Alaaeldin Aboueldahab, ${ }^{2}$ Anas Souid, ${ }^{2}$ Andrew Gunn, ${ }^{2}$ Yufeng Li, $^{5} \mathrm{Zhixin} \mathrm{Wang}^{5}$ \\ and Ammar Almehmi ${ }^{6}$ \\ ${ }^{1}$ Department of Diagnostic and Interventional Imaging, University of Texas Health Science Center at Houston, Houston, \\ TX 77030, USA \\ ${ }^{2}$ Department of Radiology, University of Alabama at Birmingham, Birmingham, AL 35233, USA \\ ${ }^{3}$ Department of Radiology, University of Arkansas for Medical Sciences, Little Rock, AR 72205, USA \\ ${ }^{4}$ Department of Radiology, Zagazig University, Zagazig, Egypt \\ ${ }^{5}$ Department of Medicine, Division of Preventive Medicine, University of Alabama at Birmingham, Birmingham, AL 35233, USA \\ ${ }^{6}$ Department of Medicine, Division of Nephrology, University of Alabama at Birmingham, Birmingham, AL 35233, USA
}

Correspondence should be addressed to Ahmed Kamel Abdel Aal; ahmed.k.abdelaal@uth.tmc.edu

Received 4 February 2020; Accepted 28 March 2020; Published 25 April 2020

Academic Editor: Paul Sijens

Copyright (c) 2020 Ahmed Kamel Abdel Aal et al. This is an open access article distributed under the Creative Commons Attribution License, which permits unrestricted use, distribution, and reproduction in any medium, provided the original work is properly cited.

\begin{abstract}
The aim of this study is to compare the outcomes of the elective-start versus urgent-start use of peritoneal dialysis (PD) catheters using percutaneous radiologic or laparoscopic techniques. Patients having their first peritoneal dialysis catheter placed and used between January 2005 and January 2018 were identified, and their medical records were retrospectively reviewed. Two groups were identified: elective-start $(n=211)$ and urgent-start $(n=29)$. Patient's demographics were similar between the two groups with the exception of age, which was higher in the elective-start group. The catheter complication rates and catheter removal rates at 3 and 12 months, mean days-to-first complication, mean days-to-catheter removal, and overall patient survival at 12 months were analyzed. Catheter complication rates at 3 and 12 months were similar between the two groups $(27.8 \%$ and $48.9 \%$, respectively, in the elective-start group versus $35.9 \%$ and $54.2 \%$, respectively, in the urgent-start group, $p=0.415)$. The catheter removal rates at 3 and 12 months were also similar between the two groups $(p=0.088)$. Catheter leak was higher in the urgent-start group (13.8\% versus $3.3 \%$, respectively, $p=0.011)$. There was no difference between the elective-start and the urgent-start groups in the mean days-to-first complication (95 vs $69, p=0.086$ ), mean days-to-catheter removal (145 vs 127, $p=0.757$ ), and overall patient survival at 12 months $(100 \%$ vs $97 \%, p=0.41)$. In conclusion, apart from catheter leak, there were similar rates of catheter complication and removal for PD catheter used for the elective-start compared to the urgent-start PD. Furthermore, the technique of placement did not affect the outcomes.
\end{abstract}

\section{Introduction}

Chronic kidney disease (CKD) as well as end-stage renal disease (ESRD) is prevalent throughout the world with an incidence of $13.6 \%$ and $0.14 \%$, respectively [1]. More than 25,000 patients in the United States receive dialysis via peritoneal dialysis (PD) [2]. PD is a patient-centered costeffective modality compared to hemodialysis (HD) [3]. This is because PD can be carried out at home, affording unique lifestyle benefits for patients, and allowing for lower dialysis costs [4]. PD also provides preservation of kidney function [5] and improved mortality [6] when compared to HD.

It is expected that PD will be utilized at a higher rate in the United States as recent modifications by the Centers for Medicare and Medicaid Services (CMS) resulted in higher reimbursement for PD services [7]. The distinct advantages 
of PD have led to the "PD First" position by some clinicians and to consider PD for urgent dialysis commencement and not only for the elective-start dialysis settings [8-10]. The urgent-start dialysis is a term used to describe patients needing immediate dialysis initiation with no preestablished vascular or PD access, where initiation of the PD occurs within 2 weeks after catheter insertion [8]. Therefore, the urgent-start PD is an attractive option for patients presenting with ESRD without prior access preparation [11].

Nevertheless, PD in the urgent-start setting is frequently underutilized for several reasons. First, patients may have difficulty making a decision regarding a dialysis modality. Second, there may be limited experience with PD or a lack of resources at multiple levels, leading to reluctance in using PD especially in patients with severe uremic symptoms and volume overload. Third, the urgent-start $\mathrm{PD}$ requires prompt PD catheter placement (typically within 24 to 48 hours of presentation), which might not be available in all institutions and medical facilities $[12,13]$. While PD catheters are better in terms of overall morbidity and mortality, they are not entirely without risk. Abdominal wall complications, most commonly hernia and leak, may occur owing to the increased intra-abdominal pressure from high dialysate volume infused in the peritoneal cavity [14]. Technical failure from malpositioned catheters may also occur [15]. Data regarding the outcomes of the elective-start compared to the urgent-start PD is limited, with few published studies available in the literature [16-18].

The purpose of this study was to compare the outcomes of PD catheter placement in the elective-start versus urgentstart settings.

\section{Methods}

2.1. Study Population. This study was approved by the local institutional review board, and informed consent was waived. A total of two hundred and sixty patients who underwent PD catheter placement between January 2005 and January 2018 were retrospectively reviewed. Two groups were identified: the elective-start group, which comprised of patients who had PD catheters inserted as part of patient preparation for dialysis access, and, the urgent-start group, which included patients who presented with no preestablished access and had their PD catheters placed for the immediate initiation of dialysis. PD catheter insertion was performed either by an interventional radiologists and nephrologists using percutaneous, image-guided technique (fluoroscopy and ultrasound guidance) using conscious sedation, or by surgeons using laparoscopic technique using general anesthesia. The decision to perform percutaneous image-guided versus laparoscopic placement of the catheters was mainly based on physician discretion taking into consideration the age and comorbidity of the patients, and the ability to perform general anesthesia. Older patients with comorbid conditions who were not eligible to obtain general anesthesia were referred for percutaneous catheter placement.

Some of the study subjects may overlap with another study titled "Outcomes of fluoroscopic and ultrasoundguided placement versus laparoscopic placement of peritoneal dialysis catheters," which was published in the Clinical Kidney Journal [19]. The objective of that study was to compare the fluoroscopy and ultrasound guidance technique with the laparoscopic technique. This study focuses on comparing the outcomes of PD catheter placement in the elective-start versus urgent-start settings regardless of the technique.

Patients with stage- 5 CKD or with ESRD, age $\geq 19$ years, and with PD catheter placed during the study period were the inclusion criteria. Exclusion criteria were patients with no documentation of follow-up at our institution or those with a PD catheter buried under the skin and not used during the study period due to the patients who did not meet the criteria for dialysis (embedded catheters). Patients who underwent laparoscopic adhesiolysis, hernia repair, or omentopexy, patients who had previous abdominal surgery, or patients with severe obesity, defined as BMI of 30 or higher, were included in both groups. Figure 1 illustrates the algorithm of including and excluding patients. Two hundred and forty patients were included in the study.

The demographics and comorbidities of the patients were obtained from the medical records. BMI was classified into 3 categories: Class- 1 is BMI of 30 to $<35$, Class- 2 is BMI of 35 to $<40$, and class- 3 is BMI of 40 or higher. We also recorded the prior surgeries of the patients.

There were 211 patients in the elective-start group as well as 29 patients in the urgent-start group. Patients in both groups had similar demographics and medical comorbidities except for age, which was higher in the elective-start group (55.8 years, $\mathrm{SD}=15.6$ ) compared to the urgent-start group (47.2 years, $\mathrm{SD}=15.9)(p=0.0094)$ (Table 1$)$. The electivestart group consisted of 121 (57.3\%) females and $90(42.7 \%)$ males, while the urgent-start group included 16 (55.2\%) males and $13(44.8 \%)$ females. Obesity was similar in the elective-start and urgent-start groups and was seen in 73 patients $(34.6 \%)$ and 12 patients $(41.3 \%)$, respectively $(p=0.47)$. The mean BMI was also similar between the elective-start group (29.2) and the urgent-start group (28.4) $(p=0.855)$. In the elective-start group, 32 patients $(15.2 \%)$, 26 patients $(12.3 \%)$, and $15(7.1 \%)$ were class- 1 , class-2, and class-3 obesity, respectively. In the urgent-start group, 8 patients $(27.6 \%), 3$ patients $(10.3 \%)$, and 1 patient $(3.4 \%)$ were class-1, class-2, and class-3 obesity, respectively. Prior surgical procedures were performed more commonly in the elective-start group (117 patients, 55.5\%) compared to the urgent-start group (10 patients, $34.5 \%)(p=0.034)$ (Table 2).

2.2. Study Outcomes. Following PD catheter placement, the patients were followed up regularly by their nephrologists. Information on catheter complication and removal was obtained from the nephrology clinic notes in the patients' medical records.

The occurrence of PD catheter-related complications at 3 and 12 months was a composite endpoint that included infectious, mechanical, and technical complications. The infectious complications included the following: exit-site infection and tunnel infection as well as peritonitis. Peritonitis was defined as an infectious process that starts at the exit site, migrates along the subcutaneous tunnel, and ends in peritonitis. The 


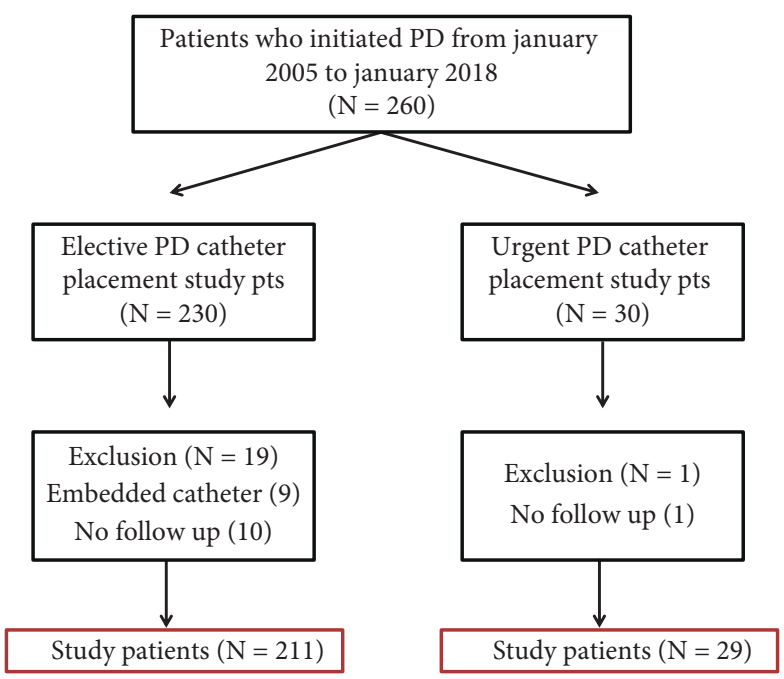

FIgURE 1: Algorithm for patients' inclusion and exclusion.

TABle 1: Demographics and comorbidities of the elective-start and urgent-start groups.

\begin{tabular}{|c|c|c|c|}
\hline Characteristics & Elective-start group, $N=211(\%)$ & Urgent-start group, $N=29(\%)$ & $p$ value \\
\hline Age* $^{*}$ & $55.8(15.6)$ & $47.2(15.9)$ & 0.009 \\
\hline Sex & & & 0.203 \\
\hline Female & $121(57.3)$ & $13(44.8)$ & \\
\hline Male & $90(42.7)$ & $16(55.2)$ & \\
\hline Body Mass index (BMI)* & $29.2(7.4)$ & $28.4(6.8)$ & 0.855 \\
\hline Diabetes & $100(47.4)$ & $15(51.8)$ & 0.662 \\
\hline Hypertension & $193(91.5)$ & $29(100)$ & 0.102 \\
\hline Coronary artery disease & $53(25.1)$ & $6(20.7)$ & 0.604 \\
\hline Congestive heart failure & $52(24.6)$ & $5(17.2)$ & 0.380 \\
\hline Peripheral vascular disease & $26(12.3)$ & $3(10.3)$ & 0.759 \\
\hline Cerebrovascular disease & $13(6.2)$ & $1(3.5)$ & 0.559 \\
\hline
\end{tabular}

${ }^{*}$ Mean (Standard deviation).

mechanical complications included the following: catheter leakage through the exit-site, catheter malfunction caused by insufficient catheter drainage, and the development of abdominal hernia. The technical complications included the following: bowel perforation, muscle hematoma, intraperitoneal bleeding, and insertion failure resulting from either failing to insert the catheter or inability to use it after successful insertion. Comparison between radiologic and laparoscopic techniques in each of the two groups was performed.

Catheter removal at 3 and 12 months (catheter removal), mean days-to-first complication, mean days-to-catheter removal, and overall patient survival were calculated. All information regarding the catheter's placement, complications, and removal were acquired from the patients' electronic medical records.

\subsection{Radiologic and Laparoscopic Technique of PD Catheter} Placement. Percutaneous PD catheter insertion utilizing image-guided technique (fluoroscopy and ultrasound) has been described in the literature $[4,20,21]$. The laparoscopic technique has likewise been described in the literature [22]. In the urgent-start group, dialysis was started within 48 hours of catheter insertion with low-volume dialysis until wound healing occurred. In the elective-start group, dialysis was started with large volume after complete wound healing, which typically occurs within 21 days.

2.4. Statistical Analysis. Descriptive analysis was presented for patient demographics, clinical history, imaging, and laboratory data, including mean, standard deviation, count, and percentage. To compare continuous variables between the two groups, two sample $t$-tests were conducted. Careful attention was given to the normality assumption, which was examined by normal probability plots and histograms. To compare categorical variables, Fisher's exact test was conducted. Time to complication and time to catheter removal were analyzed using the Kaplan-Meier (KM) method. The 3 and 12 months complication-free and catheter removal rates were estimated from KM curves. Time to complication and time to catheter removal were calculated from the date of procedure to the date of complication and the date of catheter removed within 12 months. Right censor was considered if patients lost at the end of the study or if the event did not occur within the study duration. For all 
TABLE 2: Prior surgical procedures in the elective-start and urgent-start groups.

\begin{tabular}{lcc}
\hline Type of surgery & Elective-start group, $N=211(\%)$ & Urgent-start group, $N=29(\%)$ \\
\hline Total no. of surgeries & $117(55.5)$ & $10(34.5)$ \\
Abdominal exploration & $4(1.9)$ & $0(0)$ \\
Appendectomy & $10(4.7)$ & $1(3.4)$ \\
Caesarean section & $26(12.3)$ & $2(6.9)$ \\
Cholecystectomy & $29(13.7)$ & $0(0)$ \\
Colon surgery & $3(1.4)$ & $0(0)$ \\
Fundoplication & $1(0.5)$ & $0(0)$ \\
Gastric bypass & $4(1.9)$ & $0(0)$ \\
Hernia repair & $21(10)$ & $2(6.9)$ \\
Hysterectomy & $40(19)$ & $1(3.4)$ \\
Kidney transplantation & $18(8.5)$ & $3(10.3)$ \\
Myomectomy & $1(0.5)$ & $0(0)$ \\
Nephrectomy & $13(6.2)$ & $1(3.4)$ \\
Salpingo-oophorectomy & $7(3.3)$ & $1(3.4)$ \\
Tubal ligation & $13(6.2)$ & $0(0)$ \\
\hline
\end{tabular}

inferences, the significant level was set to $p \leq 0.05$. All analyses were conducted using SAS v.9.4 (SAS Inc., USA).

\section{Results}

Catheter complication rates are shown in Table 3. In both groups, the most common complications were catheter malfunction and peritonitis accounting for $28.4 \%$ and $15.1 \%$, respectively, in the elective-start group compared to $17.2 \%$ and $27.6 \%$, respectively, in the urgent-start group but without a significant difference $(p=0.203$ and $p=0.092$, respectively). Catheter leakage was more frequent in the urgent-start group when compared to the elective-start group (13.8\% versus $3.3 \%$, respectively, $p=0.011)$. Bowel injury resulting in perforation was documented in one of the patients in the elective-start group and was placed using the image-guided technique. This patient was treated conservatively using prophylactic antibiotic administration.

Kaplan-Meier analysis revealed nonsignificant difference in the rates of catheter complications when comparing the two groups $(p=0.415)$ (Figure 2). The estimated catheter complication rates at 3 and 12 months from the KM analysis were $27.8 \%$ and $48.9 \%$, respectively, in the electivestart group versus $35.9 \%$ and $54.2 \%$, respectively, in the urgent-start group (Table 4). When patients were compared based on the technique of catheter placement (radiologic versus laparoscopic) in each group separately, there was no difference in the rate of catheter complications between the two techniques ( $p=0.834$ and $p=0.264$ for the electivestart and urgent-start groups, respectively) (Figures 3(a) and 3(b)). No difference was noted in the mean days-to-first complication which was 95 days ( $\mathrm{SD}=88$, range $0-350$ days) in the elective-start group compared to 69 days $(\mathrm{SD}=97$, range 1-300 days) in the urgent-start group $(p=0.086)$.

Kaplan-Meier analysis demonstrated no difference in the overall catheter removal rates when comparing the two groups $(p=0.088)$ (Figure 4$)$. The estimated catheter removal rates from the KM analysis are shown in Table 4 . The overall catheter removal at 3 and 12 months were $7.3 \%$ and $26.9 \%$, respectively, in the elective-start group versus $7.7 \%$ and $34.8 \%$, respectively, in the urgent-start group. There was no difference in the mean days-to-catheter removal, which was 145 days ( $\mathrm{SD}=95$, range $0-338$ days) in the elective-start group compared to 127 days ( $\mathrm{SD}=79$, range $14-235$ days) in the urgent-start group $(p=0.757)$.

Kaplan-Meier survival analysis revealed no difference in the overall survival of the patients when comparing the two groups $(p=0.41)$ (Figure 5$)$, although the mean age was higher in the elective-start group. The urgent-start group had no estimated deaths at 3 and 12 months compared to $1.0 \%$ and $3.33 \%$, respectively, in the elective-start group. Four patients died in the first year in the elective-start group, and the causes of death were not related to the procedure or catheter complications, but were due to septic shock from pneumonia in one patient, multiorgan failure in one patient, and cardiopulmonary arrest in two patients.

\section{Discussion}

PD has several advantages over HD including an improved lifestyle for patients and enhanced outcomes [23, 24]. Survival superiority of PD over HD in the first two-years of therapy has been shown, with similar outcomes up to five years [25-29]. Typically, the late-referred patients who are good candidates for PD are usually started on HD using a temporary central venous catheter due to the inability to place PD catheter in a timely manner [16]. However, temporary central venous catheters are usually associated with increased risk of complications including but not limited to sepsis and bacteremia requiring hospitalization and catheter malfunction requiring catheter replacement [30].

PD catheter placement utilizing image-guided percutaneous methods is essential for rapid commencement of PD in late-presenting patients [31]. Various centers have established pathways for surgical and image-guided percutaneous PD catheter placements to provide rapid initiation of $\mathrm{PD}$, in which assisted $\mathrm{PD}$ treatments are provided by dedicated PD nursing staff in the outpatient setting until clinical improvement, and then, the patient is trained on self-care at home [31-33]. 
TABLE 3: Catheter complication rates for the elective-start and urgent-start groups.

\begin{tabular}{lccc}
\hline & Elective-start group, $N=211(\%)$ & Urgent-start group, $N=29(\%)$ & $p$ value \\
\hline Exit site infections & $11(5.2)$ & $1(3.5)$ & 0.683 \\
Peritonitis & $32(15.1)$ & $8(27.6)$ & 0.092 \\
Catheter malfunction & $60(28.4)$ & $5(17.2)$ & 0.203 \\
Catheter leak & $7(3.3)$ & $4(13.8)$ & 0.011 \\
Primary leak & $2(1)$ & $1(3.5)$ & 0.256 \\
Hernia & $9(4.3)$ & $3(10.3)$ & 0.159 \\
Muscle hematoma or bleeding & $6(2.8)$ & $0(0)$ & 0.358 \\
Bowel perforation & $1(0.5)$ & $0(0)$ & 0.710 \\
\hline
\end{tabular}

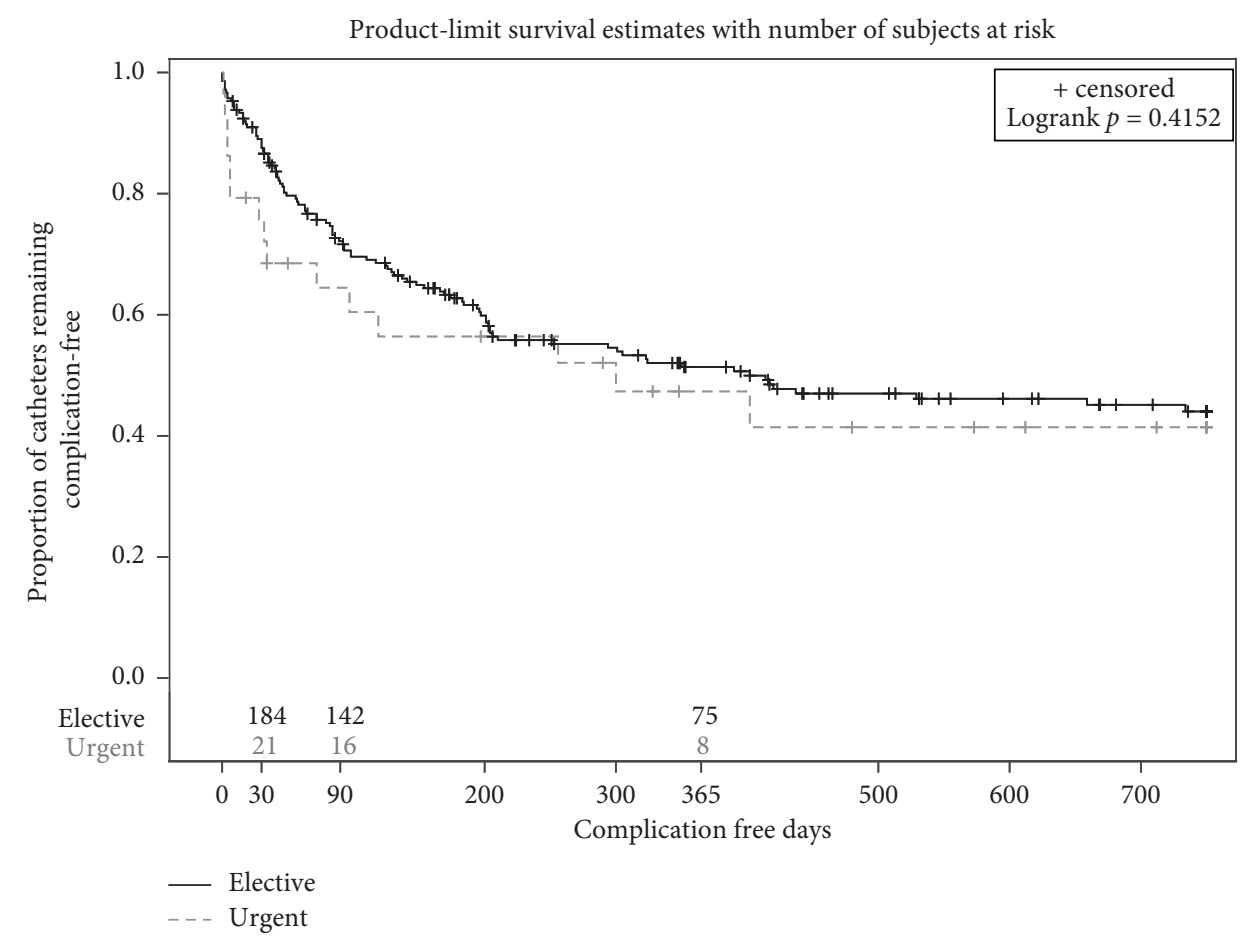

FIGURE 2: Kaplan-Meier curves for catheter complication rates in the elective-start and urgent-start groups, showing no difference between both groups.

TABLE 4: Estimated catheter complications and removal rates for the elective-start and urgent-start groups from the Kaplan-Meier curves.

\begin{tabular}{lccc}
\hline & Elective-start group, $N=211(\%)$ & Urgent-start group, $N=29(\%) p$ value $^{*}$ \\
\hline Catheter complication rate at 3 months & 27.8 & 35.9 & 0.415 \\
Catheter complication rate at 12 months & 48.9 & 54.2 & 7.7 \\
Catheter removal at 3 months & 7.3 & 34.8 \\
Catheter removal at 12 months & 26.9 & 0.088 \\
\hline
\end{tabular}

${ }^{*}$ Logrank test.

Rapid placement of PD catheters with rapid initiation of dialysis allows for a single procedure to provide both shortterm and long-term access. However, these patients frequently find themselves faced with the risk of dialysate leak by earlier use of the PD catheter $[9,16]$. In the urgent-start setting, catheter leak is one of the most commonly encountered complications ranging $0-33.3 \%$ [9, 33-39]. Our study is consistent with the published literature showing a similar risk of leak with a rate of $13.8 \%$ for the urgent-start group, which was higher than that of the elective-start group. Several maneuvers have been described to minimize the risk of dialysate leak associated with PD catheter placement in the urgent-start setting. These include recumbent dialysis only with lower dwell volumes and avoidance of dialysate dwells while the patient is upright [9]. In the elective-start setting, patients typically initiate PD after $2-3$ weeks to allow 


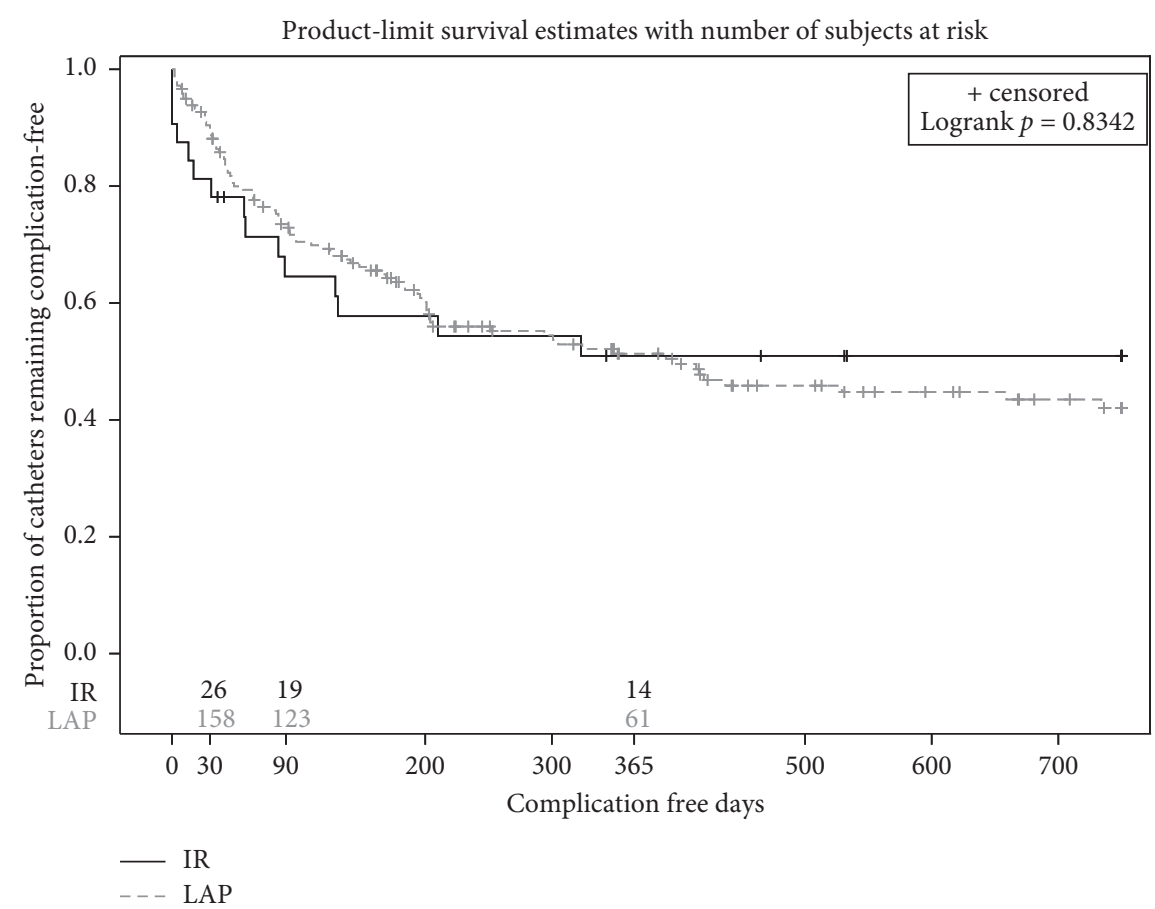

(a)

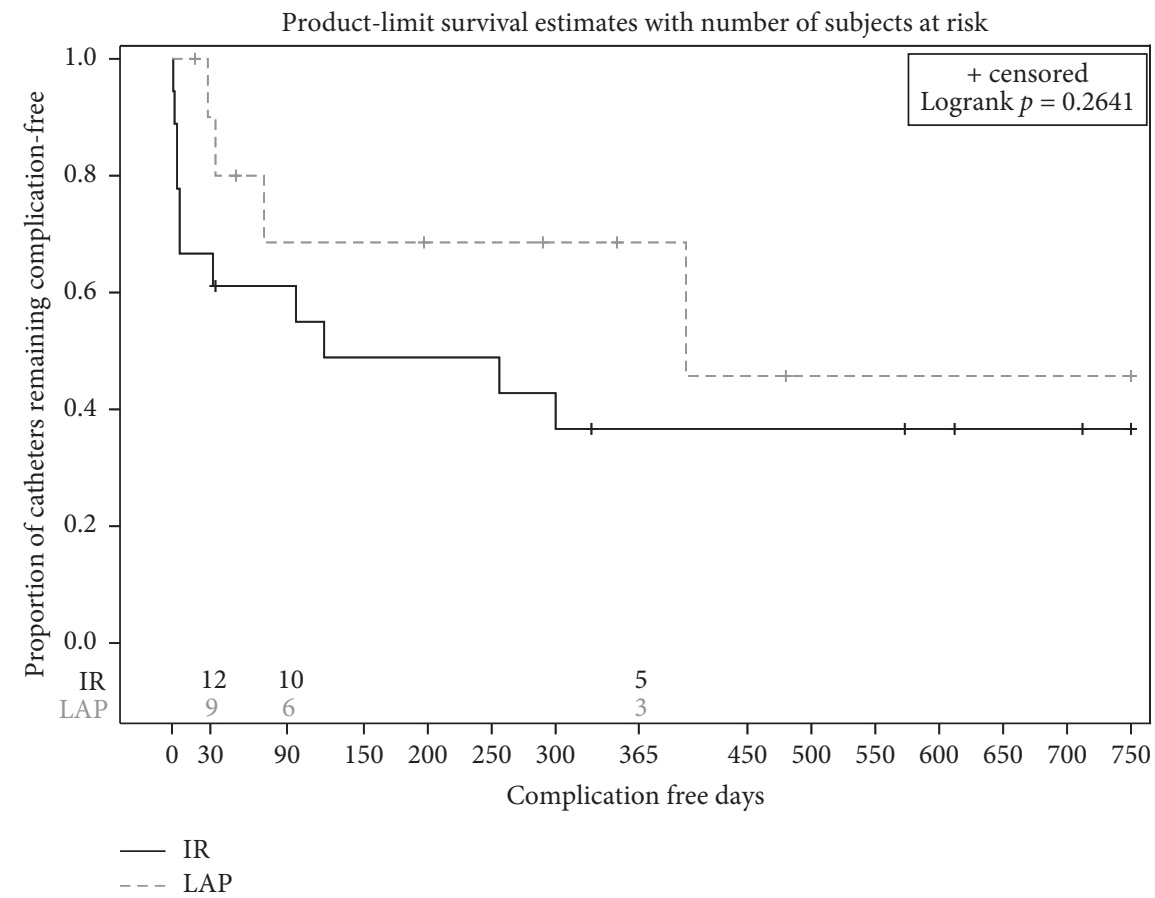

(b)

FIGURE 3: Kaplan-Meier curves for catheter complication rates based on radiologic and laparoscopic techniques in the (a) elective-start group and (b) urgent-start group. There was no difference in the complication rates based on the technique used for catheter placement.

for tissue ingrowth of the deeper Dacron cuff, which significantly minimize the risk of dialysate leak into the subcutaneous tissue [9].

The complication-free catheter survival or catheter removal rates in each group did not differ based on the technique of PD catheter placement used. Nayak et al. [16] conducted a prospective case-controlled study on 56 patients comparing the outcomes of patients who underwent conventional PD fourteen days after catheter insertion with those who underwent the urgent-start automated PD within 48 hours of catheter placement. Exit-site leak, catheter blockage, and peritonitis at 90 days were similar in both 


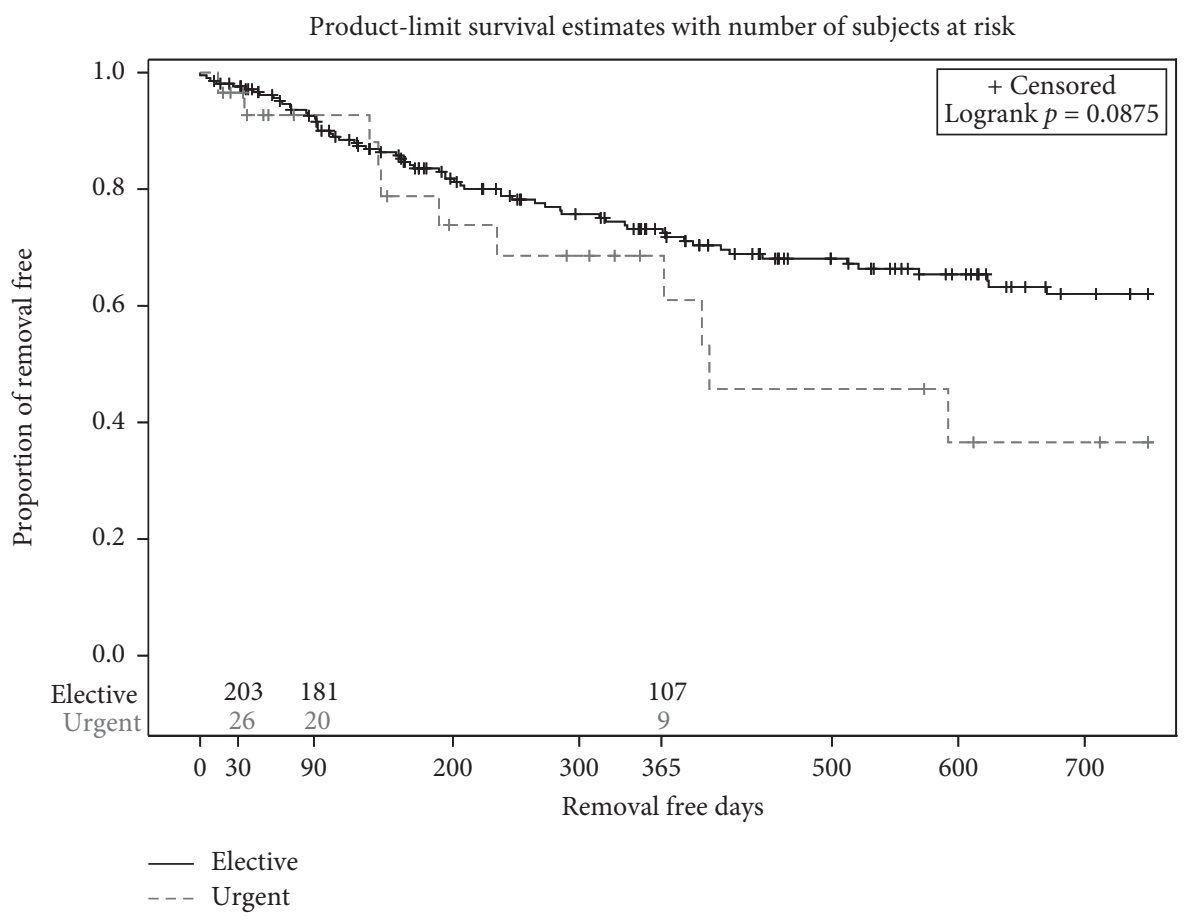

FIgURE 4: Kaplan-Meier curves for overall catheter removal in the elective-start and urgent-start groups showing no difference between the two groups.

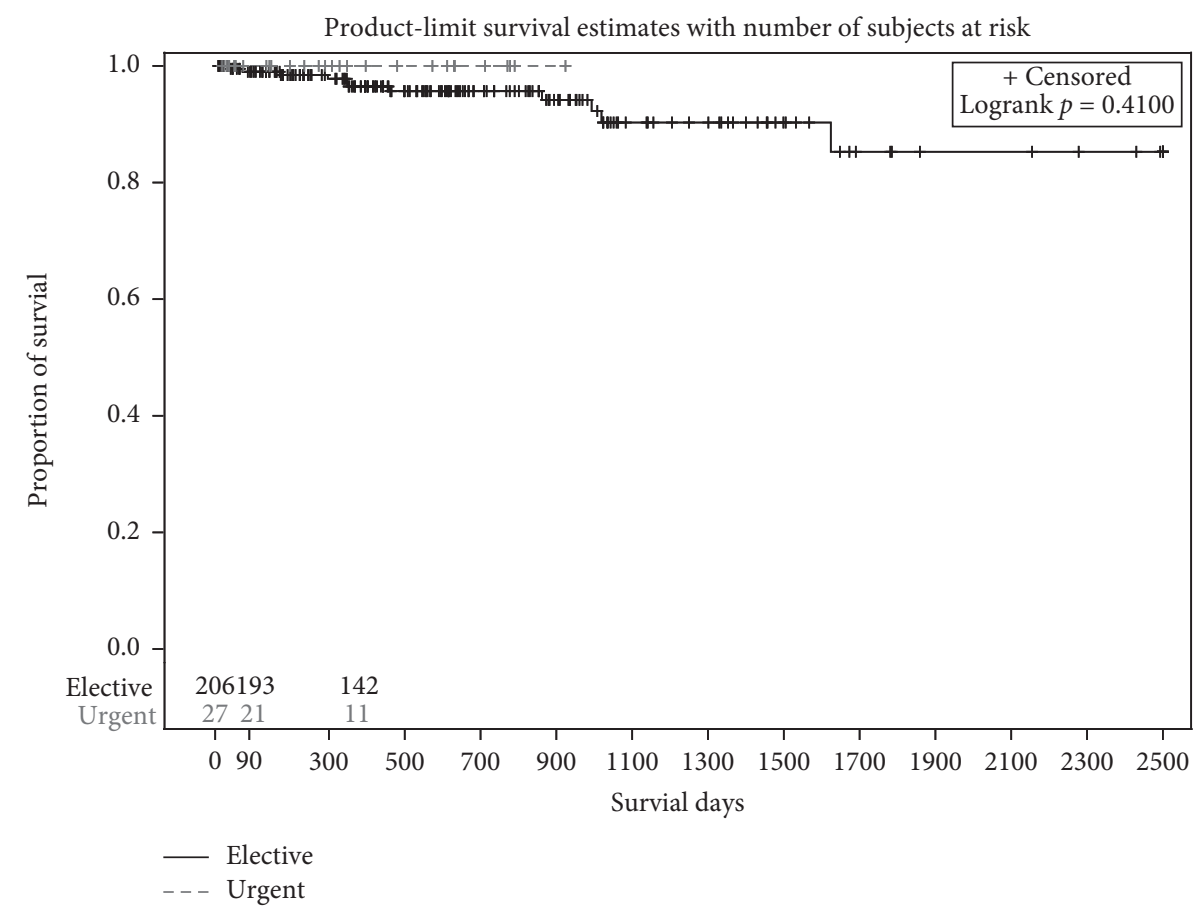

Figure 5: Kaplan-Meier curves for overall patient survival in the elective-start and urgent-start groups showing no difference in survival at 12 months.

groups, and the study concluded that urgent-start automated $\mathrm{PD}$ in the unplanned patient is an appropriate approach. Another recent study evaluated the safest and shortest interval to commence PD after catheter insertion [17]. Patients were randomized to 3 groups in which PD was started at 1 week, 2 weeks, and 4 weeks. Catheter leak was significantly higher in patients who initiated PD at 1 and 2 weeks compared to 4 weeks. The usage of manual exchanges in that study could have been responsible for the increased incidence of leaks. 
A single-center study assessed 30 patients who started on urgent PD, which was initiated within 2 weeks of catheter insertion (range: $0-13$ days, median $=6$ days), with $66.7 \%$ of the PD catheters inserted percutaneously versus 33.3\% inserted laparoscopically. Minor pericatheter leak developed in $10 \%$ of the patients during the first week of training was treated conservatively. Catheter migration resulting in dysfunction was seen in $20 \%$ of patients and was treated by repositioning, without any need for catheter exchange or change in dialysis modality. The study concluded that urgent-start PD is safe and a reasonable alternative option to hemodialysis for patients who need to start PD urgently with no preexisting dialysis access [34]. Several other retrospective studies supported the concept that shorter break-in periods are not associated with more catheter-related complications $[9,35,36]$.

Comparison between radiologic and laparoscopic techniques for placement of peritoneal dialysis catheters has been previously published in the literature [19], with no significant difference in the safety and outcomes between the two techniques. This study adds to the evolving literature signifying that PD catheter placement in the urgent-start setting, regardless of the technique used for catheter placement whether radiologic or laparoscopic, is not inferior compared to placement in the elective-start settings and therefore may offer late-referred ESRD patients an alternative patient-centered dialysis option, while avoiding the side effects of placing a CVC.

Future directions will probably focus on continued improvement in the insertion techniques to prevent leaks, implementing protocols that address the urgent-start PD needs to facilitate patient transition to outpatient home dialysis training, and increasing the awareness of the urgent-start PD program to combat the deficits seen among the ER physicians, nephrologists, hospitalists, nursing staff, and discharge planners. The success of an urgent-start program depends on the institution of a multidisciplinary approach to ensure rapid initiation and proper monitoring of PD therapy and smooth transition to long-term PD therapy. This requires teamwork of dedicated interventional radiologists and surgeons, PD nurses, renal nutritionists, and the ancillary hospital resource staff including pharmacy [16].

Limitations of this current study include its retrospective design with selection-bias being an inherent disadvantage. In addition, the study describes a single-center experience, and the results cannot be generalized. Clinical outcomes such as duration of hospital stay and mortality were not included in the analysis.

In conclusion, apart from the higher incidence of catheter leak, the outcomes of PD catheters in the urgentstart setting are not inferior to the elective-start setting with similar complication-free catheter survival and catheter removal rates at 3 and 12 months regardless of the technique used for catheter placement. These findings suggest that urgent-start PD is as safe as elective-start PD and has the advantage of avoiding the side effects and complications associated with CVC placement in late-referred ESRD patients who are considered for PD.

\section{Abbreviations and acronyms}

\author{
CKD: Chronic kidney disease \\ ESRD: End-stage renal disease \\ PD: Peritoneal dialysis \\ HD: Hemodialysis \\ CMS: Centers for Medicare and Medicaid Services \\ KM: Kaplan-Meier.
}

\section{Data Availability}

The data used to support the findings of this study are available from the corresponding author upon request.

\section{Conflicts of Interest}

Ahmed Kamel Abdel Aal: consultant: St Jude Medical, Boston Scientific, Bard Peripheral Vascular, Baxter Healthcare, Sirtex medical, Abbott Vascular, Eisai Medical, Guerbet, W. L. Gore, Surefire Medical, and Terumo Medical. Unrestricted grants from: BTG, Sirtex Medical, Bard peripheral vascular, Medtronic, and Boston Scientific. Research grants: Boston Scientific. Andrew Gunn: consultant and speaker: BTG. Speaker: Terumo, Angiodynamics. Research support: Penumbra.

\section{References}

[1] United States Renal Data System, "USRDS 2014 annual data report: atlas of chronic kidney disease and end-stage renal disease in the United States," in Ch. 1: CKD in the general population, National Institutes of Health, National Institute of Diabetes and Digestive and Kidney Diseases, Bethesda, MD, USA, 2014.

[2] United States, Department of Health and Human Services, National Institutes of Health, USRDS 2010 Annual Data Report: Atlas of Chronic Kidney Disease and End-Stage Renal Disease in the United States, National institute of diabetes and digestive and kidney diseases, US renal data system (USRDS), Bethesda, MD, USA, 2010.

[3] S. W. Klarenbach, M. Tonelli, B. Chui, and B. J. Manns, "Economic evaluation of dialysis therapies," Nature Reviews Nephrology, vol. 10, no. 11, pp. 644-652, 2014.

[4] A. K. Abdel-aal, P. Dybbro, P. Hathaway, S. Guest, M. Neuwirth, and V. Krishnamurthy, "Best practices consensus protocol for peritoneal dialysis catheter placement by interventional radiologists," Peritoneal Dialysis International: Journal of the International Society for Peritoneal Dialysis, vol. 34, pp. 481-493, 2014.

[5] C. R. Blagg, "Dialysis composite rate bundling: potential effects on the utilization of home hemodialysis, daily and nocturnal hemodialysis, and peritoneal dialysis," Seminars in Dialysis, vol. 24, no. 6, pp. 674-677, 2011.

[6] K. Yeates, N. Zhu, E. Vonesh, L. Trpeski, P. Blake, and S. Fenton, "Hemodialysis and peritoneal dialysis are associated with similar outcomes for end-stage renal disease treatment in Canada," Nephrology Dialysis Transplantation, vol. 27, no. 9, pp. 3568-3575, 2012.

[7] T. A. Golper, S. Guest, J. D. Glickman, J. Turk, and J. P. Pulliam, "Home dialysis in the new USA bundled payment plan: implications and impact," Peritoneal Dialysis International: Journal of the International Society for Peritoneal Dialysis, vol. 31, no. 1, pp. 12-16, 2011. 
[8] K. Chaudhary, H. Sangha, and R. Khanna, "Peritoneal dialysis first: rationale," Clinical Journal of the American Society of Nephrology, vol. 6, no. 2, pp. 447-456, 2011.

[9] A. Ghaffari, "Urgent-start peritoneal dialysis: a quality improvement report," American Journal of Kidney Diseases, vol. 59, no. 3, pp. 400-408, 2012.

[10] R. Arramreddy, S. Zheng, A. B. Saxena, S. E. Liebman, and L. Wong, "Urgent-start peritoneal dialysis: a chance for a new beginning," American Journal of Kidney Diseases, vol. 63, no. 3, pp. 390-395, 2014.

[11] M. J. Schreiber Jr., "Changing landscape for peritoneal dialysis: optimizing utilization," Seminars in Dialysis, vol. 30, no. 2, pp. 149-157, 2017.

[12] L. M. Moist and C. E. Lok, "Incident dialysis access in patients with end-stage kidney disease: what needs to Be improved," Seminars in Nephrology, vol. 37, no. 2, pp. 151-158, 2017.

[13] A. Ghaffari, V. Kumar, and S. Guest, "Infrastructure requirements for an urgent-start peritoneal dialysis program," Peritoneal Dialysis International: Journal of the International Society for Peritoneal Dialysis, vol. 33, no. 6, pp. 611-617, 2013.

[14] J. M. Bargman, "Complications of peritoneal dialysis related to increased intraabdominal pressure," Kidney International Supplements, vol. 40, pp. S75-S80, 1993.

[15] B. B. McCormick and J. M. Bargman, "Noninfectious complications of peritoneal dialysis: implications for patient and technique survival: table 1,"Journal of the American Society of Nephrology, vol. 18, no. 12, pp. 3023-3025, 2007.

[16] K. S. Nayak, S. V. Subhramanyam, N. Pavankumar, S. Antony, and M. A. Sarfaraz Khan, "Emergent start peritoneal dialysis for end-stage renal disease: outcomes and advantages," Blood Purification, vol. 45, no. 4, pp. 313-319, 2018.

[17] D. Ranganathan, G. T. John, E. Yeoh et al., "A randomized controlled trial to determine the appropriate time to initiate peritoneal dialysis after insertion of catheter (Timely PD study)," Peritoneal Dialysis International: Journal of the International Society for Peritoneal Dialysis, vol. 37, no. 4, pp. 420-428, 2017.

[18] E. J. See, Y. Cho, C. M. Hawley, L. R. Jaffrey, and D. W. Johnson, "Early and late patient outcomes in urgentstart peritoneal dialysis," Peritoneal Dialysis International: Journal of the International Society for Peritoneal Dialysis, vol. 37, no. 4, pp. 414-419, 2017.

[19] A. K. Abdel Aal, S. S. Guest, S. Moawad et al., "Outcomes of fluoroscopic and ultrasound-guided placement versus laparoscopic placement of peritoneal dialysis catheters," Clinical Kidney Journal, vol. 11, no. 4, pp. 549-554, 2018.

[20] A. K. Abdel Aal, S. Gaddikeri, and S. Saddekni, "Technique of peritoneal catheter placement under fluoroscopic guidance," Radiology Research and Practice, vol. 2011, Article ID 141707, 4 pages, 2011.

[21] A. K. Abdel-Aal, A. K. Joshi, S. Saddekni, and I. D. Maya, "Fluoroscopic and sonographic guidance to place peritoneal catheters: how we do it," American Journal of Roentgenology, vol. 192, no. 4, pp. 1085-1089, 2009.

[22] E. Maher, M. J. Wolley, S. A. Abbas, S. P. Hawkins, and M. R. Marshall, "Fluoroscopic versus laparoscopic implantation of peritoneal dialysis catheters: a retrospective cohort study," Journal of Vascular and Interventional Radiology, vol. 25, no. 6, pp. 895-903, 2014.

[23] A. W. Yu, K. F. Chau, Y. W. Ho, and P. K. Li, "Development of the "peritoneal dialysis first" model in Hong Kong," Peritoneal Dialysis International, vol. 27, no. 2, pp. S53-S55, 2007.

[24] P. K.-T. Li and C.-C. Szeto, "Success of the peritoneal dialysis programme in Hong Kong," Nephrology Dialysis Transplantation, vol. 23, no. 5, pp. 1475-1478, 2008.
[25] E. F. Vonesh, J. J. Snyder, R. N. Foley, and A. J. Collins, "The differential impact of risk factors on mortality in hemodialysis and peritoneal dialysis," Kidney International, vol. 66, pp. 2398-2401, 2004.

[26] J. G. Heaf, H. Løkkegaard, and M. Madsen, "Initial survival advantage of peritoneal dialysis relative to haemodialysis," Nephrology Dialysis Transplantation, vol. 17, no. 1, pp. 112117, 2002.

[27] S. S. A. Fenton, D. E. Schaubel, M. Desmeules et al., "Hemodialysis versus peritoneal dialysis: a comparison of adjusted mortality rates," American Journal of Kidney Diseases, vol. 30, no. 3, pp. 334-342, 1997.

[28] B. G. Jaar, J. Coresh, L. C. Plantinga et al., "Comparing the risk for death with peritoneal dialysis and hemodialysis in a national cohort of patients with chronic kidney disease," Annals of Internal Medicine, vol. 143, no. 3, pp. 174-183, 2005.

[29] E. D. Weinhandl, R. N. Foley, D. T. Gilbertson, T. J. Arneson, J. J. Snyder, and A. J. Collins, "Propensity-matched mortality comparison of incident hemodialysis and peritoneal dialysis patients," Journal of the American Society of Nephrology, vol. 21, no. 3, pp. 499-506, 2010.

[30] H. S. Vats, "Complications of catheters: tunneled and nontunneled," Advances in Chronic Kidney Disease, vol. 19, no. 3 , pp. 188-194, 2012.

[31] F. X. Liu, A. Ghaffari, H. Dhatt et al., "Economic evaluation of urgent-start peritoneal dialysis versus urgent-start hemodialysis in the United States," Medicine, vol. 93, no. 28, p. e293, 2014.

[32] A. Masseur, S. Guest, and V. Kumar, "Early technique success after initiation of treatment with urgent-start peritoneal dialysis," Advances in Peritoneal Dialysis, vol. 30, no. 30, pp. 36-39, 2014.

[33] A. Casaretto, R. Rosario, W. R. Kotzker, Y Pagan-Rosario, C Groenhoff, and S Guest, "Urgent-start peritoneal dialysis: report from a U.S. private nephrology practice," Advances in Peritoneal Dialysis, vol. 28, no. 28, pp. 102-105, 2012.

[34] A. M. A. Alkatheeri, P. G. Blake, D. Gray, and A. K. Jain, "Success of urgent-start peritoneal dialysis in a large Canadian renal program," Peritoneal Dialysis International: Journal of the International Society for Peritoneal Dialysis, vol. 36, no. 2, pp. 171-176, 2016.

[35] Y.-F. Yang, H.-J. Wang, C.-C. Yeh, H.-H. Lin, and C.-C. Huang, "Early initiation of continuous ambulatory peritoneal dialysis in patients undergoing surgical implantation of tenckhoff catheters," Peritoneal Dialysis International: Journal of the International Society for Peritoneal Dialysis, vol. 31, no. 5, pp. 551-557, 2011.

[36] J. V. Povlsen and P. Ivarsen, "How to start the late referred ESRD patient urgently on chronic APD," Nephrology Dialysis Transplantation, vol. 21, no. 2, pp. ii56-ii59, 2006.

[37] J. H. Song, G. A. Kim, S. W. Lee, and M.-J. Kim, "Clinical outcomes of immediate full-volume exchange one year after peritoneal catheter implantation for CAPD," Peritoneal Dialysis International: Journal of the International Society for Peritoneal Dialysis, vol. 20, no. 2, pp. 194-199, 2000.

[38] O. Banli, H. Altun, and A. Oztemel, "Early start of CAPD with the Seldinger technique," Peritoneal Dialysis International: Journal of the International Society for Peritoneal Dialysis, vol. 25, no. 6, pp. 556-559, 2005.

[39] Y. Jo, S. K. Shin, J. Lee, J. Song, and J. Park, "Immediate initiation of CAPD following percutaneous catheter placement without break-in procedure," Peritoneal Dialysis International: Journal of the International Society for Peritoneal Dialysis, vol. 27, no. 11, pp. 179-183, 2007. 